\title{
Routine immunisation services in Nepal during the COVID-19 pandemic
}

\section{Mathema S ID}

Smriti Mathema, Associate Professor, Department of Paediatrics, Kathmandu Medical College Teaching Hospital, Kathmandu, Nepal.

W ith the international public health emergency brought by the novel coronavirus disease (COVID-19) pandemic, countries initiated frantic measures to contain and reduce the transmission of the severe acute respiratory syndrome coronavirus 2 , or SARS-CoV-2. This translated into a dramatic shift of health care resources, closure of country borders, travel restrictions and implementation of physical distancing. As Nepal went into its first nationwide lockdown last year on March 24 2020, immunisation services for Nepal's 8.5 million children came to a complete halt in centres throughout the country. 'Safely resuming routine childhood immunisations became a major priority, to protect children from vaccine-preventable infections with morbidity and mortality far greater than COVID-19.

Immunisation services are cornerstones of public health preventing millions of deaths each year. Many more million lives are protected from the suffering and disability associated with diseases such as pneumonia, diarrhoea, meningitis, polio, measles, and whooping cough. The direct and societal benefits of routine childhood vaccination are huge and have an impact on the country's economy. Being one of the most cost-

Access this article online

Website: www.jkmc.com.np

DOI: https://doi.org/10.3126/jkmc.v10i1.38944

HOW TO CITE

Mathema S. Routine immunisation services in Nepal during the covid-19 pandemic. J Kathmandu Med Coll. 2021;10(1):1-3.

Address for correspondence

\section{Dr. Smriti Mathema}

Associate Professor, Department of Paediatrics

Kathmandu Medical College Teaching Hospital,

Sinamangal, Kathmandu, Nepal

E-mail: smritimathema@gmail.com

Copyright @ 2021 Journal of Kathmandu Medical College (JKMC)

ISSN: 2019-1785 (Print), 2091-1793 (Online)

(i) \& This work is licensed under a Creative Commons Attribution-Non Commercial 4.0 International License. effective health investments, it is important to prioritise strategies that make routine immunisation accessible to even the most rural and vulnerable populations. It is recognised that immunisation plays a central role in reaching the Sustainable Development Goals (SDGs), ${ }^{2}$ not only impacting directly on SDG3 (good health and well-being) but also contributing to 14 out of the 17 SDGs in reducing hunger, ending poverty and increasing equity. ${ }^{3}$ However, even before the COVID-19 pandemic, the Global Vaccine Action Plan (GVAP) report states that coverage of essential vaccines has stagnated and vaccine equity is still a challenge, both within and between countries. ${ }^{4}$ We can now but imagine how the interruption in delivery of routine immunisation caused by the pandemic is likely to lead to a secondary health crisis from disease outbreaks to amplification of economic gaps, especially in vulnerable groups of lowand middle-income countries (LMICs).

The national immunisation schedule of Nepal includes 12 antigens, protecting children against VaccinePreventable Diseases (VPDs) such as tuberculosis, polio, diphtheria, whooping cough, tetanus, hepatitis B, Haemophilus influenza borne diseases, rotaviral diarrhoea, pneumococcal diseases, measles, rubella, and Japanese Encephalitis. The National Immunisation Program (NIP) is one of the most successful programs of the Ministry of Health and Population and has reached several milestones from being certified polio free in 2014, achieving control of rubella and congenital rubella syndrome in August 2018 to attaining hepatitis B control among children in July 2019. ${ }^{5}$ In the last 47 years of its existence, this program has been able to reach up to almost $97 \%$ of target populations of $0-15$ months age group. ${ }^{5}$ Since 2002, Nepal has been getting support from the Gavi Alliance for health system strengthening, new vaccine introduction and vaccine support. There are 16,500 delivery points for fixed sessions, outreach sessions and mobile clinics in place for the delivery of immunisation services and around 7000 health workers are directly involved in providing these services. "Khoja ra Khopa " (search and immunise) is one of the most 
important initiatives and effective guiding principles that our service providers follow. Nepal is also the first country in the South East Asia region to have an Immunisation Act, therefore supporting and strengthening the NIP.

During the chaos caused by worldwide pandemics such as the current one, long term consequences of disruption of routine health care such as childhood immunisation is often overlooked. Even short periods of interference in immunisation services can result in Vaccine-Preventable Disease (VPD) outbreaks and an accumulation of susceptible individuals in the community. ${ }^{6}$ Outbreaks like these may result in excess risk of morbidity and mortality from VPD and an increased burden on an already COVID-19 strained health system. ${ }^{7}$ Like in rest of the world, Nepal too saw a decline in its routine immunisation services due to various challenges in continuity of delivery of immunisation services (Figure 1). The accessibility and supply of vaccines was poor due to transport restrictions during and after the lockdown. Furthermore, the fear of contracting the virus prevented people from travelling and visiting hospitals. Unemployment and economic hardship shifted priorities and less people proactively seeked health care. Vaccinators themselves were relocated for COVID care and many got infected, leading to a shortage of health care workers (HCWs) in immunisation clinics. There also was a struggle with availability and unequal distribution of Personal Protective Equipment (PPE). It was observed that even when immunisation services resumed at some centres, parents remained unaware due to lack of information and knowledge.

We already have seen an overall reduced rate of national immunisation coverage over the last one year. A further decline in the national coverage of several of the antigens is expected in the months to follow. Since then, we have had sporadic measles outbreaks in places like Dhading and Gorkha. We now should anticipate a reduced herd immunity against and a gradual rise in- VPDs in Nepal (Figure 2). As a result of which we should also expect a rising trend in Nepal's Infant Mortality Rate (IMR) of 32 and Under-5 Mortality Rate of 39 as per the 2016 National Demographic Health Survey (NDHS) $2016 .{ }^{8}$

Nepal has definitely taken measures to mitigate these immunisation challenges. The Department of Health Services, Ministry of Health and Population, Nepal also took out a statement in relation to recommencement of routine immunisation services and set out important guidelines, ${ }^{9}$ highlighting the need for infection control measures such as social distancing, screening and personal protection of HCWs. It was also emphasised that the target population, children between 0-15 months, should be prioritised for vaccination. Many HCWs have undergone training to adapt safety measures and caregivers have been actively communicated with about how services have been reconfigured to ensure safety.

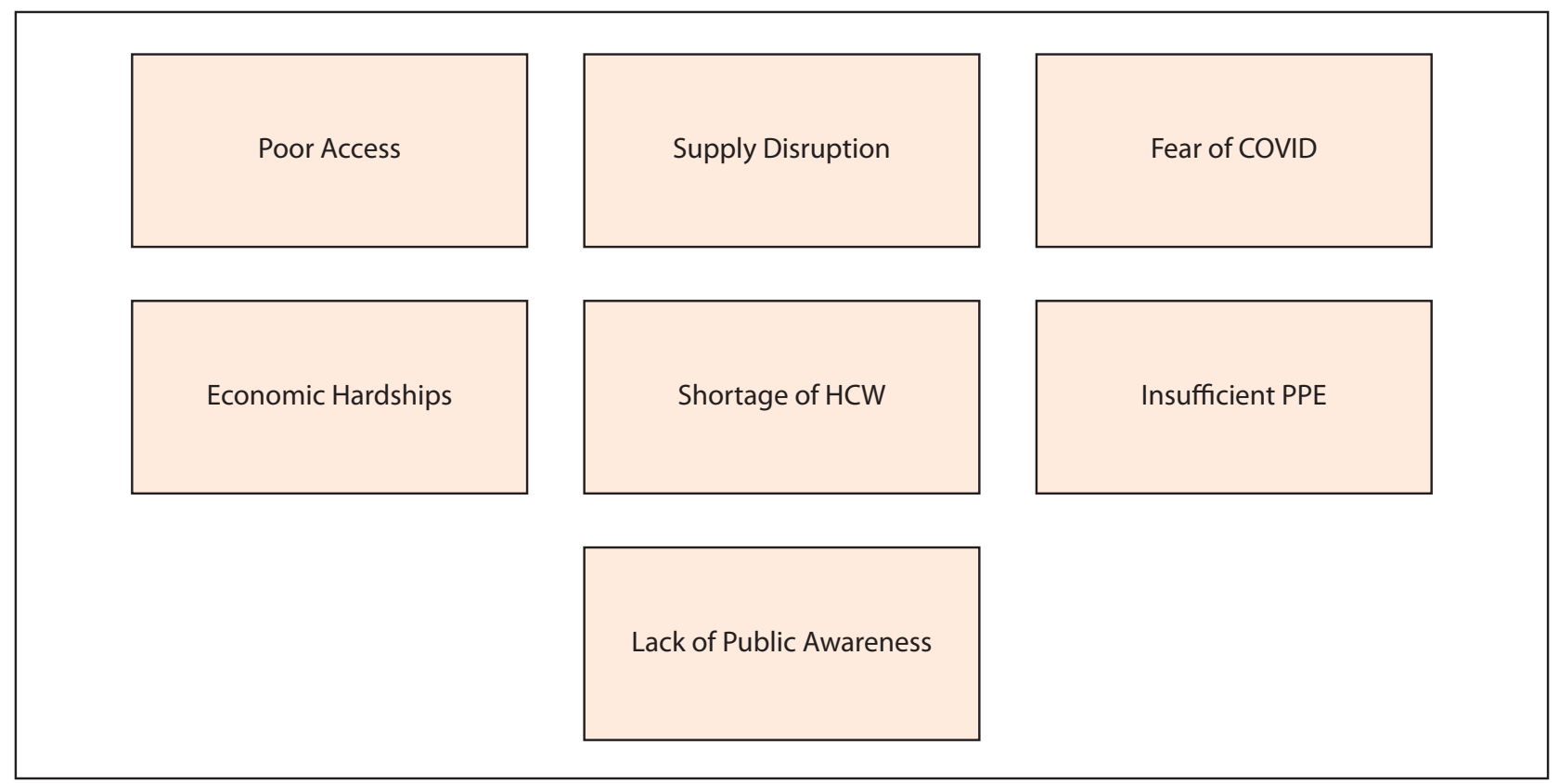

Figure 1: Barriers to immunisation during Covid-19 in Nepal 


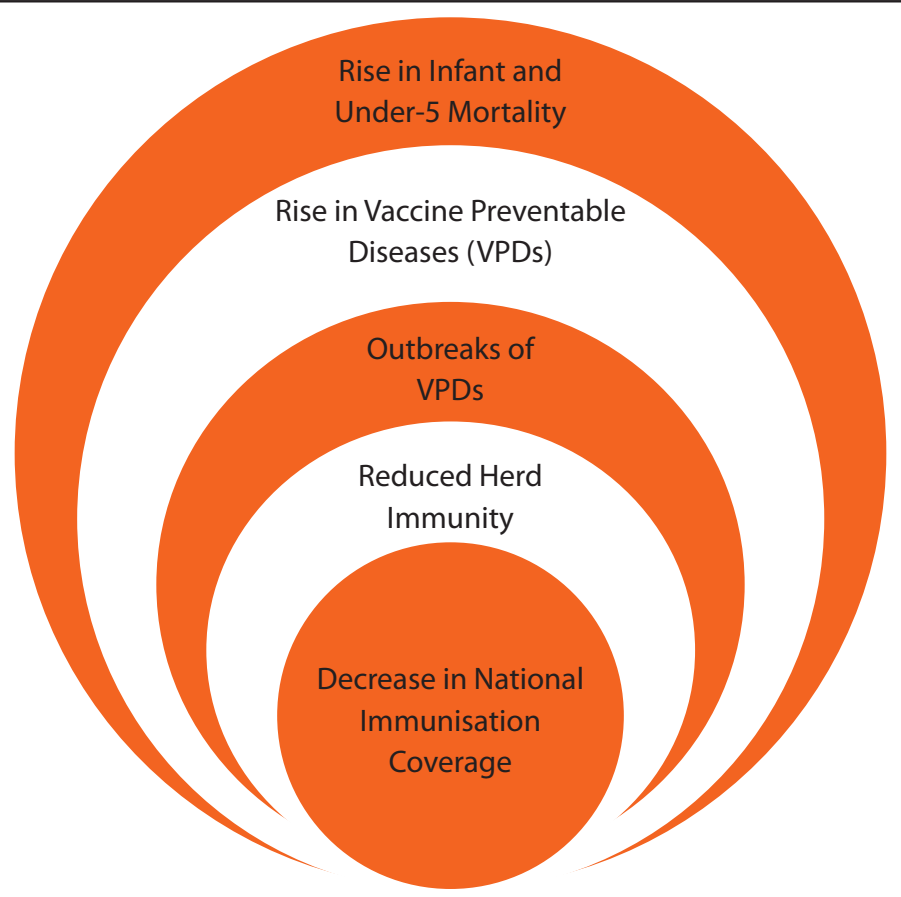

Figure 2: Impact on child health in Nepal

Required coordination has been implemented between local administration and all stakeholders. Throughout the past few months, special media campaigns urging routine childhood vaccinations have also commenced. However, in spite of the Government's direction to all provinces to resume routine immunisations, not all centres in the country have been able to provide their usual services.

\section{REFERENCES}

1. Central bureau of statistics, National Planning Commission, Government of Nepal, Central Bureau of Statistics. [Full Text]

2. United Nations General Assembly (2015). Transforming our world: The 2030 agenda for sustainable development. [Full Text]

3. Decouttere C, De Boeck K, Vandaele N. Advancing sustainable development goals through immunisation: A literature review. 2020. [Full Text]

4. Strategic Advisory Group of Experts on Immunisation. The Global vaccine action plan 20112020: Review and lessons learned. World Health Organisation; 2020. [Full Text]

5. Department of Health Services, Ministry of Health, Government of Nepal. Annual report fiscal year
In conclusion, disruptions to safe delivery of routine immunisation services caused by the COVID-19 pandemic pose a significant threat to children's health. The government's response along with multi-sectoral efforts, has partially helped restore vaccine services. Nepal should now go on a path to build back better by rectifying coverage gaps and reaching missed communities, where some of the most vulnerable children live.

2075-2076, November 2; 2020. DoHS Annual Report FY 2075-76; Department of Health Services: 2020. [Full Text]

6. Suk JE, Jimenez AP, Kourouma M, Derrough T, Balde M, Honomou $\mathrm{P}$, et al. Post-Ebola Measles Outbreak in Lola, Guinea, January-June 2015(1). Emerg Infect Dis. 2016 Jun;22(6):1 106-8. [PubMed |Full Text | DOI]

7. Guidance on routine immunisation services during COVID-19 pandemic in the WHO European Region. Copenhagen: WHO Regional Office for Europe; 2020; 1. [Full Text]

8. Nepal 2016 Demographic and Health Survey; Key Findings. The DHS Program; Nepal: 2017. [Full Text]

9. MoHP, GoN, 2020. (Subject: In Relation to Routine Immunisation Services), Circulation Letter issued by MOHP. Department of Health Services; Nepal: May 2020. 